International Journal of Modeling, Simulation, and Scientific Computing

Vol. 8, No. 4 (2017) 1750051 (15)pages)

(C) The Author(s)

DOI: $10.1142 / \mathrm{S} 1793962317500519$

\title{
Extraction, classification and visualization of 3-dimensional clouds simulated by cloud-resolving atmospheric model
}

\author{
Daisuke Matsuoka \\ Center for Earth Information Science and Technology (CEIST) \\ Japan Agency for Marine-Earth Science and Technology (JAMSTEC) \\ 3173-25 Showa-Machi, Kanazawa-ku, Yokohama 236-0001, Japan \\ daisuke@jamstec.go.jp
}

Received 8 December 2016

Accepted 20 April 2017

Published 26 May 2017

\begin{abstract}
Cloud-resolving atmospheric general circulation models using large-scale supercomputers reproduce realistic behavior of 3-dimensional atmospheric field on a global scale. To understand the simulation result for scientists, conventional visualization methods based on 2-dimensional cloud classification are not enough for understanding individual clouds and their physical characteristics. In this study, we propose a new 3-dimensional extraction and classification method of simulated clouds based on their 3-dimensional shape and physical properties. Our proposed method extracts individual clouds by cloud water and cloud ice, and classifies them into six types by their altitude and upward flow. We applied the method to time-varying atmospheric simulation data, and attempted to visualize atmospheric phenomena on the tropics such as developing cumulonimbus and tropical cyclone. Two case studies clearly visualize the behavior of individual cloud type and clarify that some cloud types have a relationship with rainfall during active weather phenomena. The proposed method has the potential to analyze such phenomena that develop in the vertical direction as well as the horizontal direction.
\end{abstract}

Keywords: Atmospheric simulation; cloud; visualization.

\section{Introduction}

Atmospheric phenomena such as a weather front, heavy rain and tropical cyclone affect the lives of human beings. Understanding these phenomena is important for social issues as well as academic issues. A large number of studies have been conducted in atmospheric and climate science fields by numerical simulations 1 The

This is an Open Access article published by World Scientific Publishing Company. It is distributed under the terms of the Creative Commons Attribution 4.0 (CC-BY) License. Further distribution of this work is permitted, provided the original work is properly cited. 
conventional global atmospheric models based on primitive equations with hydrostatic approximation are not able to resolve individual clouds. Recently, because of the advances in supercomputing technology, NICAM (Nonhydrostatic Icosahedral Atmospheric Model) ${ }^{2 / 3}$ was developed for the Earth Simulator. NICAM calculates vertical flow without hydrostatic approximation and is able to reproduce vertical convection and $\mathrm{O}(10 \mathrm{~km})$ to $\mathrm{O}(100 \mathrm{~km})$ cloud clusters and is used to analyze MJO (Madden-Julian Oscillation) ${ }^{4}$ tropical cyclones $\$ \sqrt[5]{ }$ and other global phenomena.

Generally, clouds have various types strongly related to atmospheric phenomena. To deeply understand such phenomena, understanding individual clouds and their physical properties is necessary. The latest cloud-resolving model can calculate the 3-dimensional (3D) configuration of individual clouds accurately; however, most previous studies for automatic cloud classification are intended at 2-dimensional (2D) simulation and satellite observational data i.e.,, 96 and ground-based imagery data i.e., 10111 based on 2D information such as the cloud color, top height of clouds and precipitation (amount of rainfall). Therefore, they are not adequate for understanding vertical information of individual clouds, especially their $3 \mathrm{D}$ shape and physical properties.

In this work, we propose a new extraction and classification method for 3D clouds from cloud-resolving simulation data according to their 3D shape and physical properties. Furthermore, we adopt the proposed method to visualize weather phenomena such as developing cumulonimbus and tropical cyclone on the tropical ocean. This paper is a revised version based on the conference proceedings $\frac{12}{}$ with additional case studies and detailed analysis. All case studies have been carried out with advice from scientists in the field of meteorology and climate science.

\section{Data Set}

The atmospheric simulation data used in this study is produced by the NICAM. The NICAM was first developed for the Earth Simulator and currently is also carried out on the $\mathrm{K}$ computer. The grid model uses an icosahedral grid structure and the horizontal resolution of the computational mesh is approximately $3.5 \mathrm{~km}$. This model employs fully compressible nonhydrostatic equations to obtain statistically equilibrium states and guarantees the conservation of mass and energy. Equations are discretized by finite volume method. One characteristic feature of this model is that it explicitly calculates deep convective circulations without using cumulus parameterizations. For details of the model, please see the survey paper:2

The output data includes water vapor (3D), quantities of cloud water (3D), cloud ice (3D), velocity field (3D), precipitation (2D), outgoing long radiation (OLR) (2D). A snapshot image of a simulation result is shown in Fig. 1. 2D distribution of both clouds and precipitation (hourly mean) are visualized by gray scale color and rainbow color, respectively. Several clouds and rainfall areas are reproduced on the ground as well as on the ocean. Analysis domain in Sec. 3 for method description and Sec. 4 for application are shown in squares with a red solid border. 


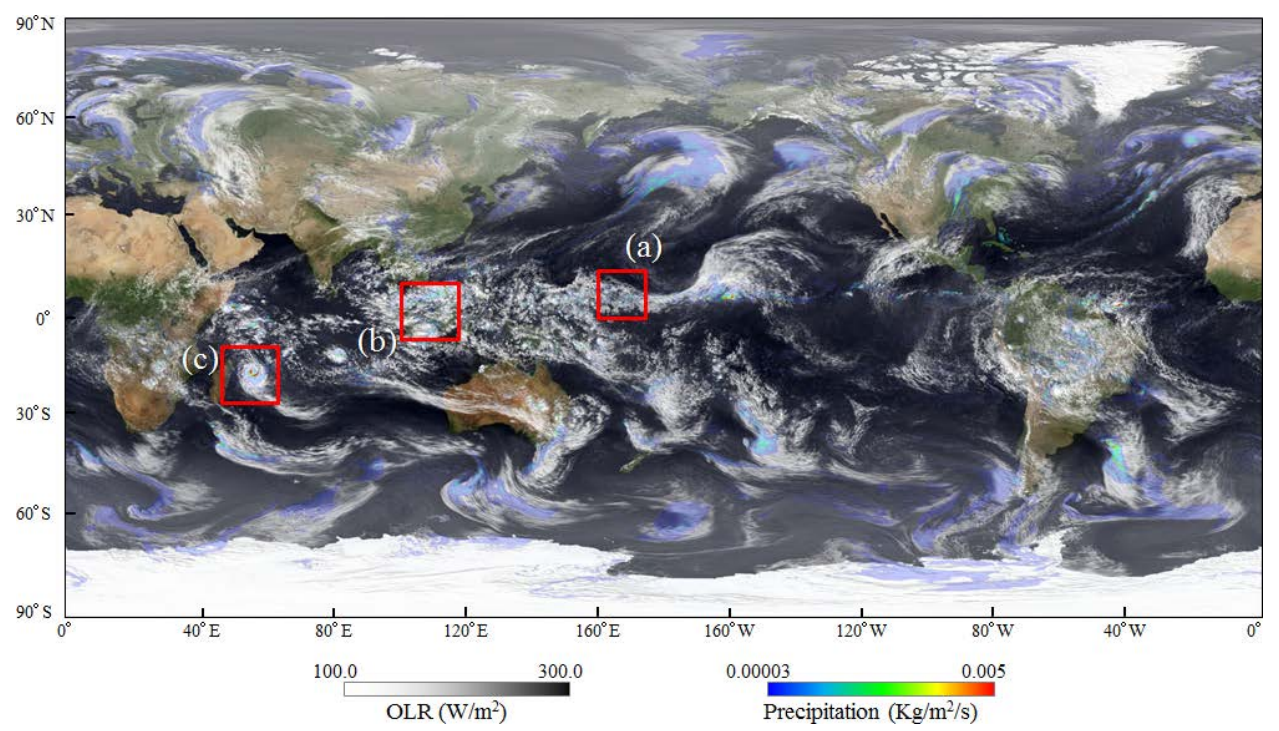

Fig. 1. A snapshot image of simulation results of OLR and precipitation (hourly mean) at 9:00:00, 12/07/2011 UTC and analysis domain of (a) the Equatorial Pacific (Sec. 3), (b) the Southeast Asia (Sec. 4.1) and (c) the Southern Indian Ocean (Sec. 4.2).

\section{Method}

\subsection{Concept of proposed method}

While real clouds have 3D configuration, previous work on cloud classification has not considered vertical distribution, as mentioned in Sec. 1 The general cloud forms have been standardized and published in the International Cloud Atlas by World Meteorological Organization (WMO) ${ }^{13}$ as shown in a basic reference for schematic cloud types in Fig. 2(a). In this reference, clouds are roughly classified into 10 types such as cirrus, stratus and cumulus based on their altitude and physical properties.

Simulated clouds by numerical model do not correspond to all cloud forms because some cloud forms cannot be reproduced by the current physical scheme and spatial resolution. Hence, this study proposes a new simple and basic 3D classification method for simulated clouds based on their altitude and upward flow. The proposed method classifies 3D clouds into the following six types, as shown in Fig. 2(b): (1) cumulonimbus, (2) cumulus, (3) low-middle clouds, (4) low clouds, (5) middle clouds and (6) high clouds. A couple of cloud forms proposed by WMO are lamped together to one group in our proposed method. For example, cirrus, cirrostratus and cirrocumulus are grouped as (6) high clouds. Similarly, altostratus and altocumulus are grouped as (5) middle clouds, and stratus, stratocumulus and nimbostratus are grouped as (4) low clouds. On the other hand, (1) cumulonimbus and high clouds can be classified using their altitude only, however, cumulus and cumulonimbus are not physically defined. Therefore, in our method, these two 


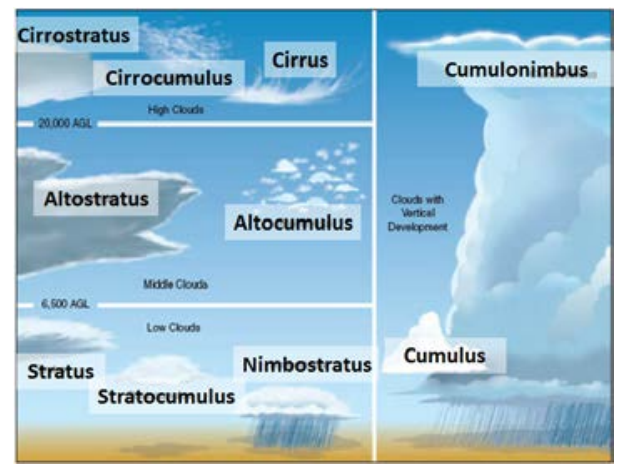

(a)

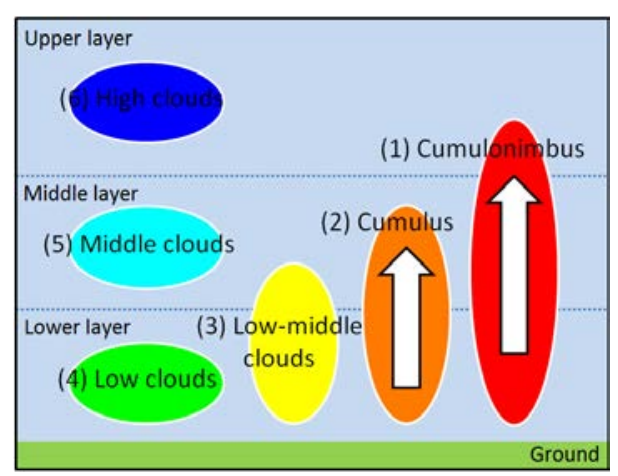

(b)

Fig. 2. Classification of clouds: (a) 10 types of cloud classification by WMC 13 and (b) a conceptual image of six types of cloud classification (proposed method).

types of clouds are classified using upward flow as well as altitude as described later. The clouds from low to middle layer which do not satisfy the condition of upward value are newly categorized as (3) low-middle clouds. Middle-high clouds are not categorized same as WMO because they are mostly cumulonimbus.

\subsection{Extraction of clouds}

A cloud is a visible mass of liquid or solid droplets made up of tiny water droplets or ice crystals, usually a mixture of both. In this study, clouds are detected from $3 \mathrm{D}$ simulation data on each grid point, where $q c+q i \geqq q t h$. Here, qc, qi and $q t h$ are quantity of cloud water, quantity of cloud ice and the threshold value for cloud detection, respectively. Individual cloud mass is defined by the spatially contiguous grid points that meet the conditions of cloud water and cloud ice. The algorithm is shown below.

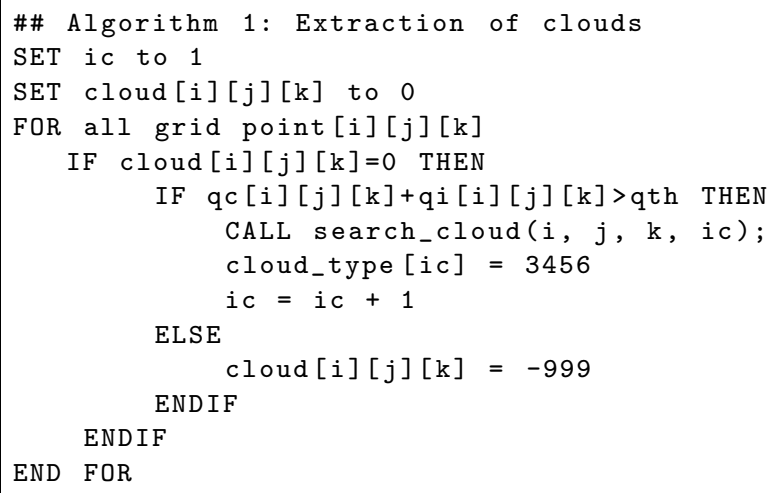




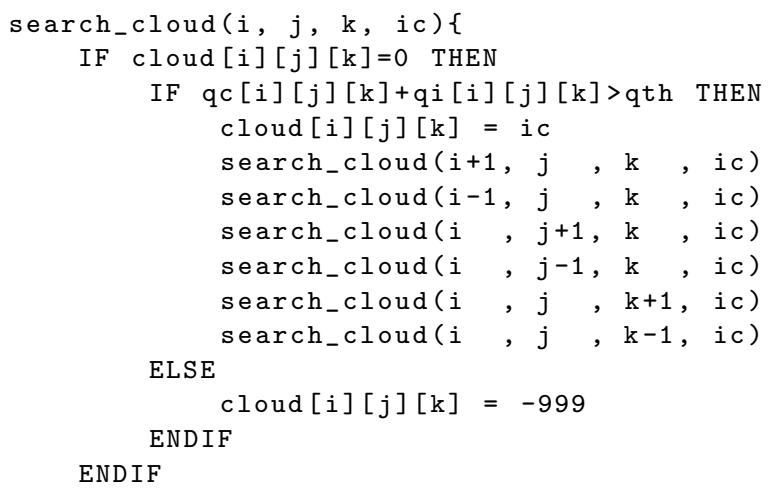

Above algorithm explores unexplored grid points to the contiguous six directions (two directions of longitude, latitude and altitude) from seed point in order to identify identical cloud. This algorithm is a recursive algorithm that repeatedly uses the subroutine search_cloud() for exploring neighboring grid points. If the currently explored grid point fulfills the condition of cloud detection, the grid point is grouped with the identical clouds and stored as the candidate for the next seed point. Repeating this recursive process, the algorithm can identify an individual cloud. cloud $[i][j][k]$ indicates an existence of a cloud on each grid point $(1,2,3, \ldots$ : cloud, 0: candidate of cloud, -999: not cloud). An integer value of 1 or more indicates the identification number ic for individual cloud mass. Figure 3(a) depicts an extraction result of 3D clouds by using the proposed method (here, $q t h=0.0001 \mathrm{~kg} / \mathrm{kg}$ ). However, Fig. 3(b) depicts $2 \mathrm{D}$ clouds visualized by OLR (same figure with Fig. 11). While both results roughly correspond with each other, the shapes of the cumulonimbus are slightly different. Cumulonimbus with vertical

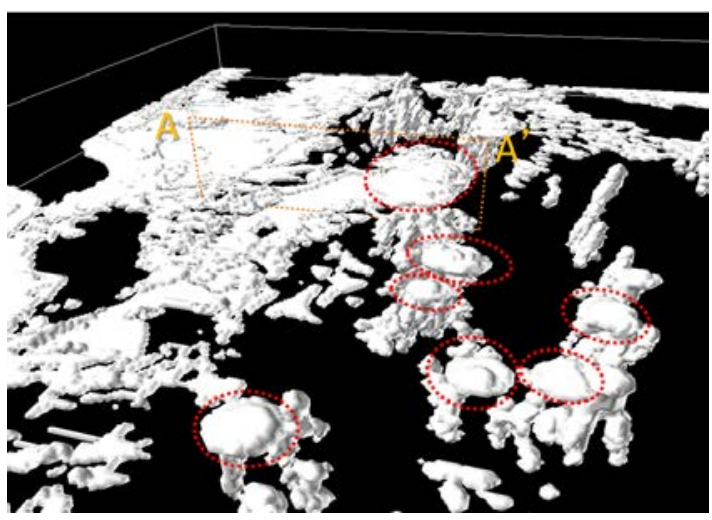

(a)

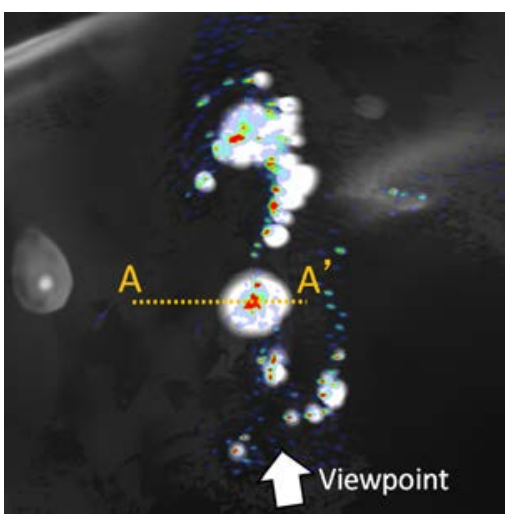

(b)

Fig. 3. Visualization results of clouds in (a) 3D (proposed method) and (b) 2D (same as Fig. 1). 


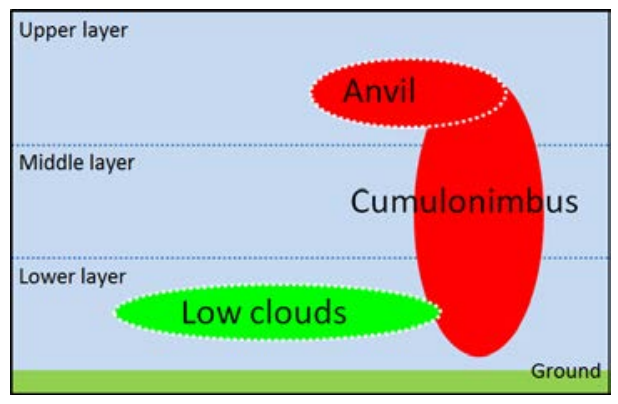

(a)

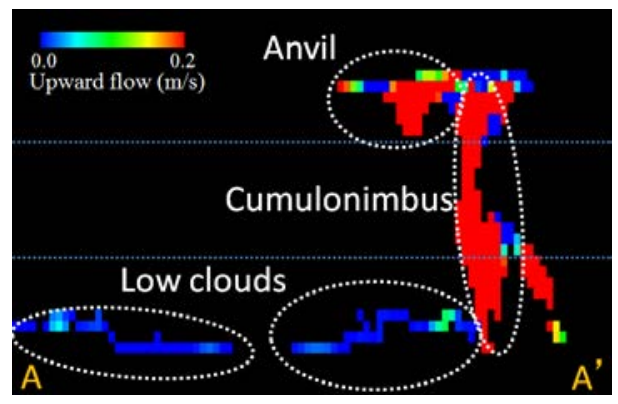

(b)

Fig. 4. Handling of connected clouds. Vertical image of (a) cloud form and (b) upward flow.

flow is known to be unable to move upward beyond the boundary of the troposphere, and it spreads widely in a horizontal direction.14 This spread cloud at the top of cumulonimbus is called an anvil or incus (red dashed line in Fig. 3(a)). While Fig. 3(b) represents the horizontal distribution of an anvil, Fig. 3. (a) visualizes 3D configurations of cumulonimbus with an anvil.

In some cases, the above-mentioned algorithm has a problem to be solved. There is a case where vertically developed clouds such as cumulonimbus and cumulus partially connect horizontally distributed clouds such as lower clouds, as shown in Fig. 4(a). Separating these essentially different clouds using an additional physical property is necessary. Figure 4(b) depicts upward flow (vertical component of velocity field) in clouds represented in Fig. 3 (yellow dashed line). While the magnitude of upward flow of the cumulonimbus is greater than $0.2 \mathrm{~m} / \mathrm{s}$ (anvil cloud is $0.0-0.2 \mathrm{~m} / \mathrm{s}$ ), the same value of the lower cloud is $0.0-0.02 \mathrm{~m} / \mathrm{s}$. Therefore, they can be separated using the threshold value of upward flow. Our proposed cloud extraction method is organized in two steps: cumulonimbus/cumulus extraction (Algorithm 2) and other clouds extraction (Algorithm 1). Algorithm 2 to extract cumulonimbus and cumulus is listed below.

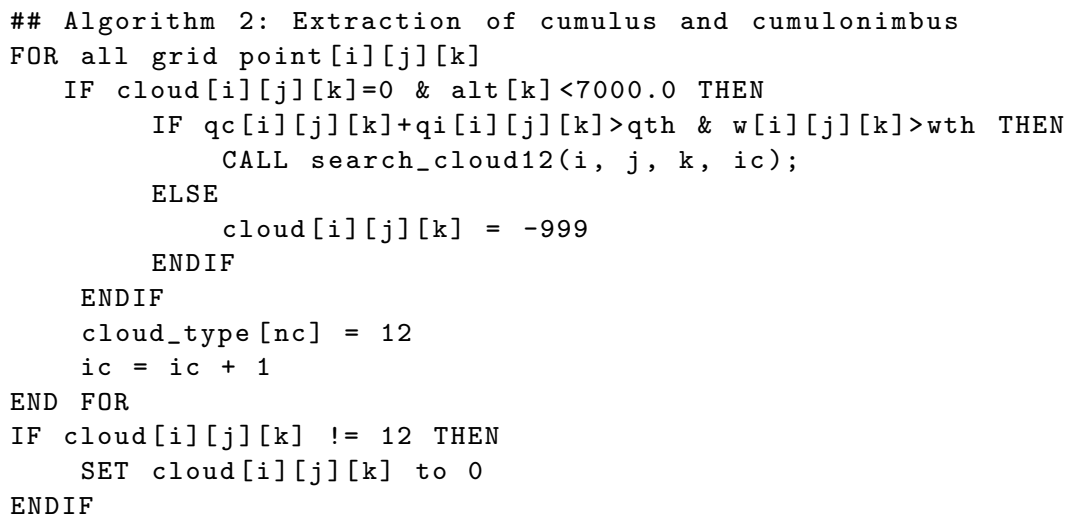




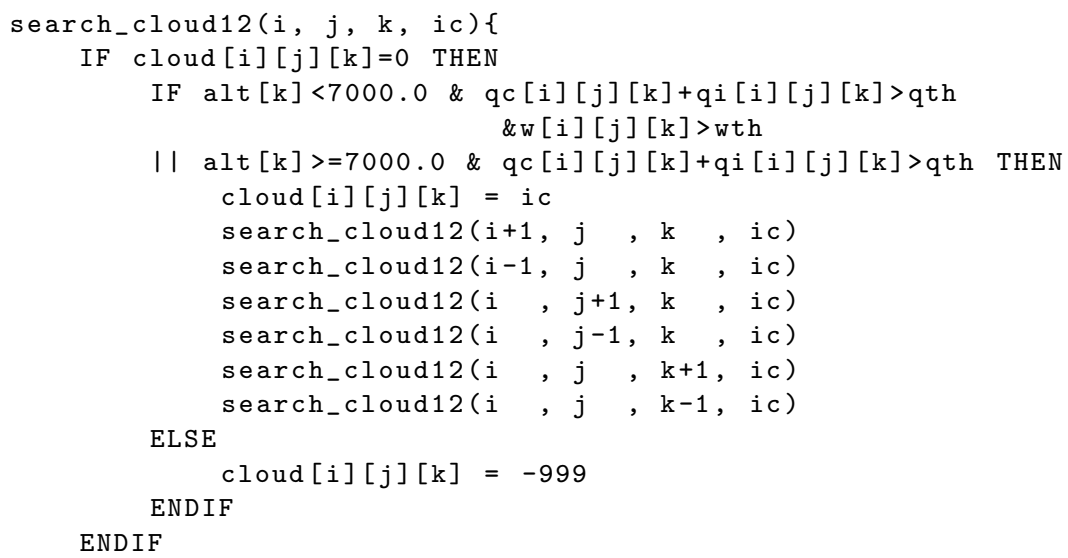

The above-listed algorithm is carried out before line 3 in Algorithm 1 . The difference between Algorithm 2 and Algorithm 1 is whether the condition of cloud extraction includes threshold value of upward flow (here, wth $=0.2 \mathrm{~m} / \mathrm{s}$ ) or not. Furthermore, in order to extract an anvil cloud, when the altitude of an explored grid point is higher than 7000.0, the threshold value wth is not included in the condition of cumulonimbus/cumulus detection. The value of cloud_type [ic], the unique ID number of the ic-th cloud, is 12 for cumulonimbus/cumulus and 3456 for other clouds. The separation result of both cloud forms is represented in Fig. 5 . Pale red and pale blue clouds indicate cumulonimbus/cumulus and other clouds,

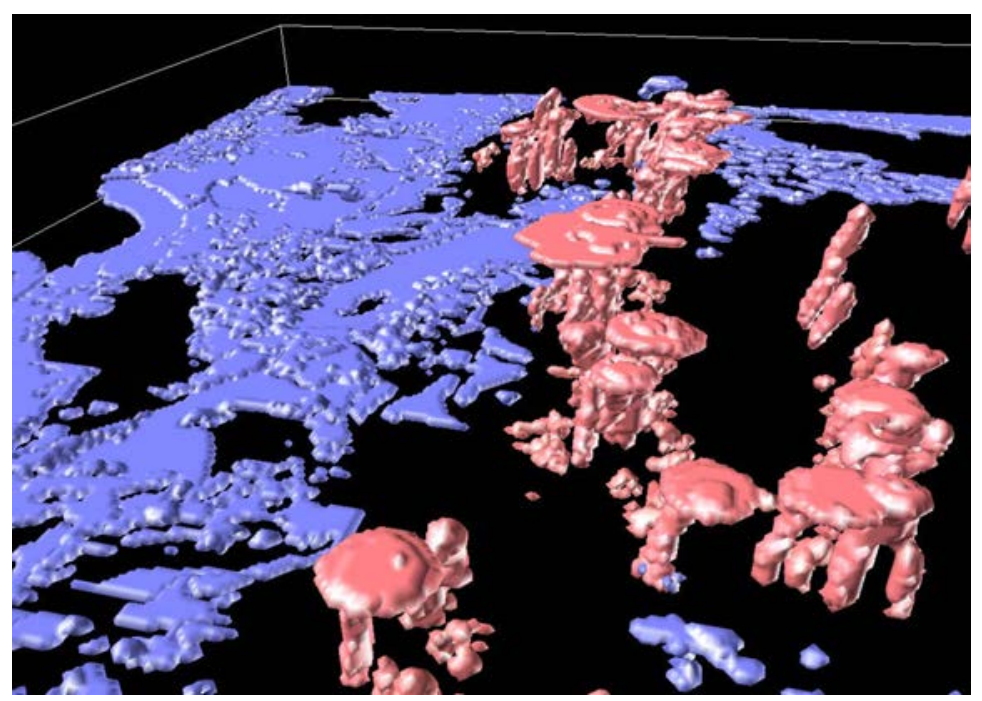

Fig. 5. Separation of cumulonimbus/cumulus and other clouds. 
respectively. This separation is a preparation for the six type cloud classification as mentioned in Sec. 3.3.

\subsection{Classification of clouds}

Detected cumulonimbus and cumulus (cloud_type [ic] =12) are classified into the following two types based on their altitude: (1) lower to upper layer and (2) lower to middle layer, respectively. In the same way, other types of detected clouds (cloud_type $[i c]=3456$ ) are classified into the following four types: (3) lower to middle layer, (4) lower layer, (5) middle layer and (6) upper layer. The algorithm is shown below.

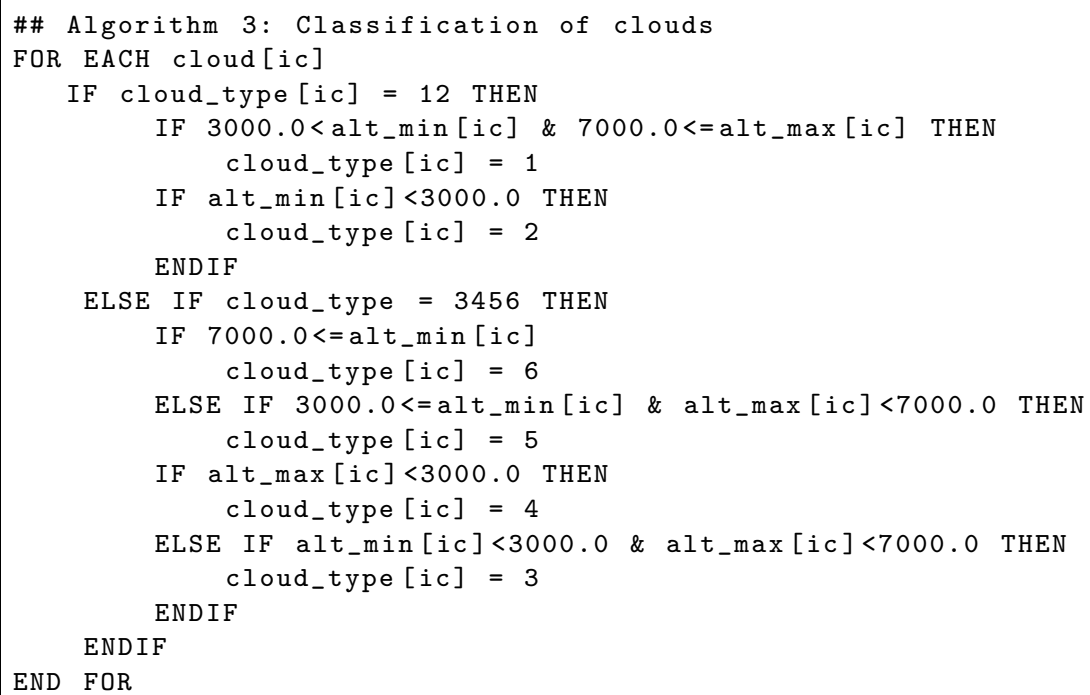

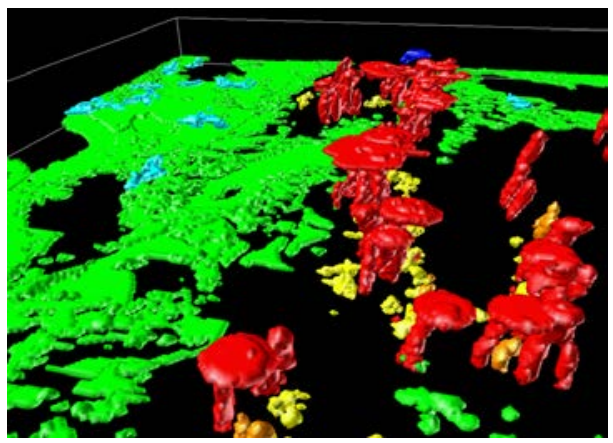

(a)

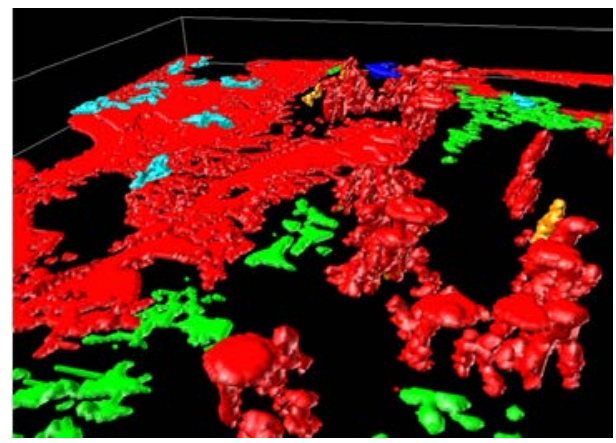

(b)

Fig. 6. Visualization results (a) using cloud extraction with cumulonimbus/cumulus separation (Algorithms 1 and 2) and cloud classification (Algorithm 3), and (b) simple cloud extraction (only Algorithm 1) and similar classification method to Algorithm 3. 
The classification results are represented in Fig. 6. Figure 6(a) is a result of using both Algorithm 1 and Algorithm 2 for cloud extraction, and Algorithm 3 for cloud classification. On the other hand, Fig. 6(b) is a result of using Algorithm 1 for cloud extraction and similar classification algorithm to Algorithm 3 based on their altitude (not completely same because the value of cloud_type [ic] is different between Algorithms 1 and 2). In both results, extracted clouds are colored by red (cumulonimbus and anvil), orange (cumulus), yellow (low-middle clouds), green (low clouds), cyan (middle clouds) and blue (high clouds), as shown in Fig. 2(b). Compared to Fig. 6(b), cumulonimbus with anvil and cumulus are correctly separated from other clouds in Fig. 6(a).

\section{Results and Discussion}

\subsection{Application to the Southeast Asia}

The proposed method is applied to visualize and analyze clouds in Southeast Asia. This area contains the South China Sea and the Southeast Asian countries as shown in Area (b) in Fig. 1. Visualization results from 13:00:00 12/02/2011 to 10:00:00 12/03/2011 LT (Local Time) are depicted in Figs. 17(a) -7(h). Low clouds are formed on the ground in the early afternoon, and low-middle clouds and cumulonimbus also occur on the land over the midnight. These clouds flow eastward (right side direction in Fig. [7). Shortly before noon, low clouds appear onshore. Cumulonimbus rarely occurs on the sea.

Next, each type of clouds is investigated from the perspective of their volume and precipitation under them. Figure $8(\mathrm{a})$ and 8 (b) show temporal change of cloud volume and precipitation from December 1 to 6, 2011 LT. From these results, the following facts are established:

- Although there are some variations from day to day, the amount of low clouds, middle clouds and high clouds changes approximately periodically. Their peak times are approximately around noon (10:00-13:00 LT), midnight-dawn (1:007:00 LT) and afternoon-evening (13:00-22:00 LT), respectively.

- Cumulonimbus dominates the majority type in all time step.

- Temporal change of precipitation also has an one-day cycle similar to the amount of cloud.

These facts agree with the paper which investigates the temporal changes in Sumatra using observational data $\frac{15}{15}$ Following facts are findings unique to the proposed method, which are not known from the analysis using observation data or 2D information.

- The temporal change of the amount of low clouds, middle clouds and high clouds also have a one-day cycle and their peak times are approximately 12:00 LT, 1:00-4:00 LT and 19:00 LT, respectively. 

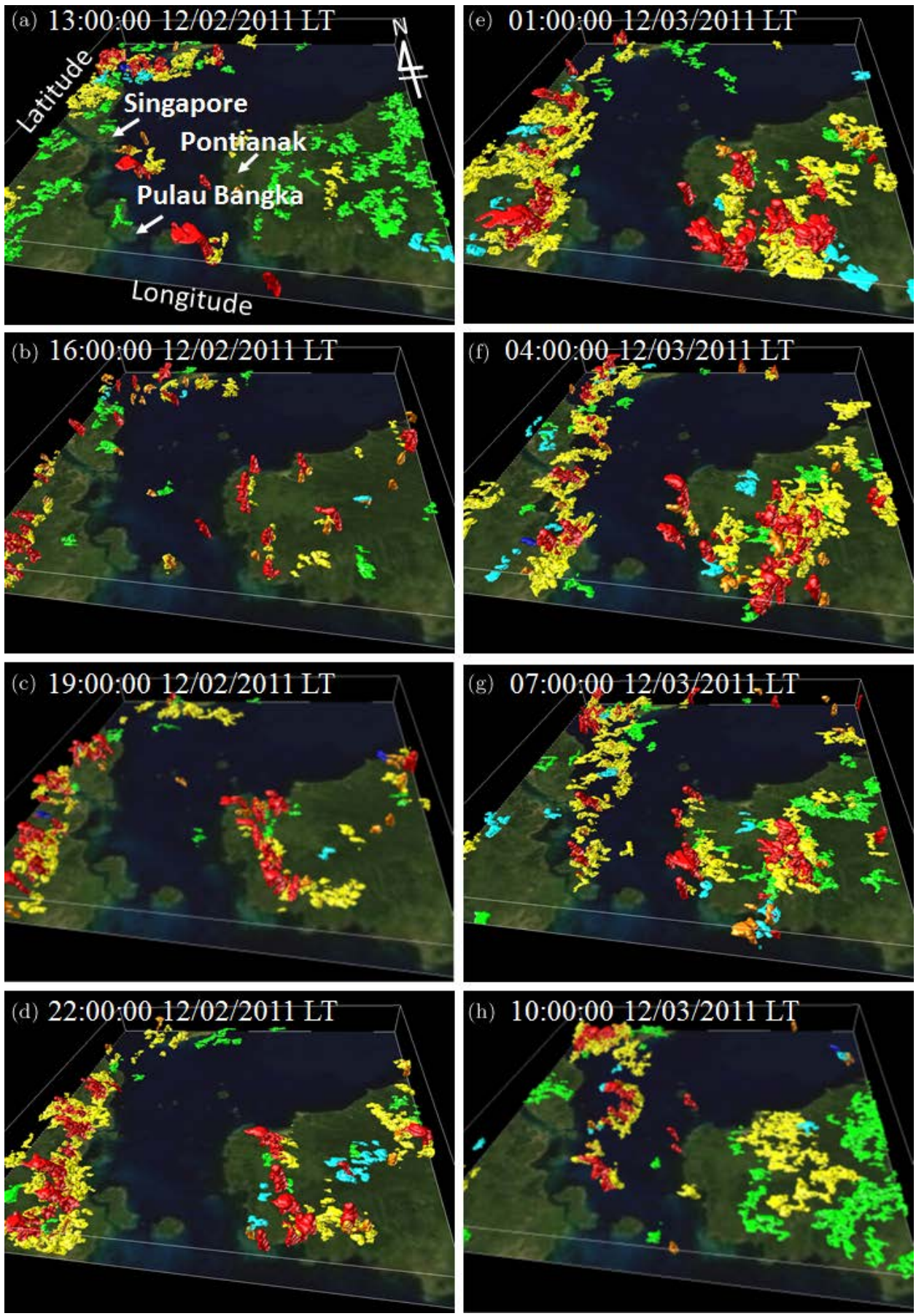

Fig. 7. Visualization results of temporal change of the clouds on Southeast Asia. 
Extraction, classification and visualization of 3-dimensional clouds

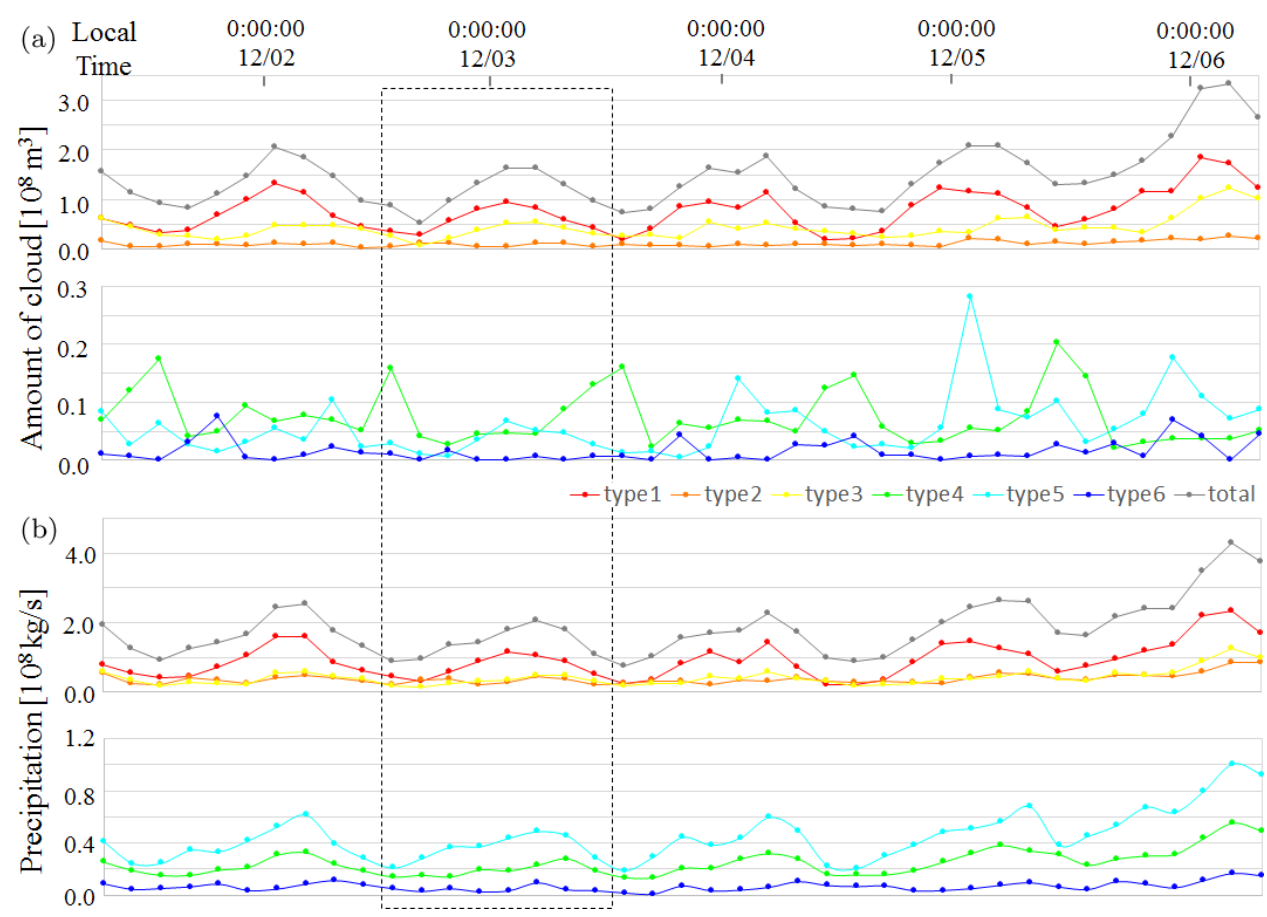

Fig. 8. Temporal change of cloud type and precipitation on the South China Sea. The Period with dotted line corresponds to visualization results (Fig. 7).

- However, their precipitation has no correlation. The precipitation value of all cloud types are large in midnight and small in daytime.

\subsection{Application to tropical cyclone}

The proposed method is applied to visualize tropical cyclones on the Indian Ocean in December 2011. Tropical cyclones are known to have swirling clouds that are vertically developed. ${ }^{1617}$ Its $2 \mathrm{D}$ (horizontal or vertical) structure has been studied, $\stackrel{17}{[17}$ however, its $3 \mathrm{D}$ structure is not understood, especially from the perspective of an individual cloud form. Although there is no clear definition of the life cycle of tropical cyclone, it is roughly classified into following four stages: initial stage, developing stage, fully developed stage, and dissipation stage.

Figure 9 displays visualization results of a tropical cyclone using the proposed six-type cloud classification during December 1-8, 2011. In the initial stage of the tropical cyclone, cumulonimbus and cumulus are developed, and low clouds and low-middle clouds gather around them (Figs. 9(a)-9(c)). In the developing stage, such clouds are swirled around the cumulonimbus, and the eye and eye wall (which is a wall of cumulonimbus around the eye) are formed (Figs. 9)(c)9(e)). This vortex structure, including swirled clouds, the eye and the eye wall, 

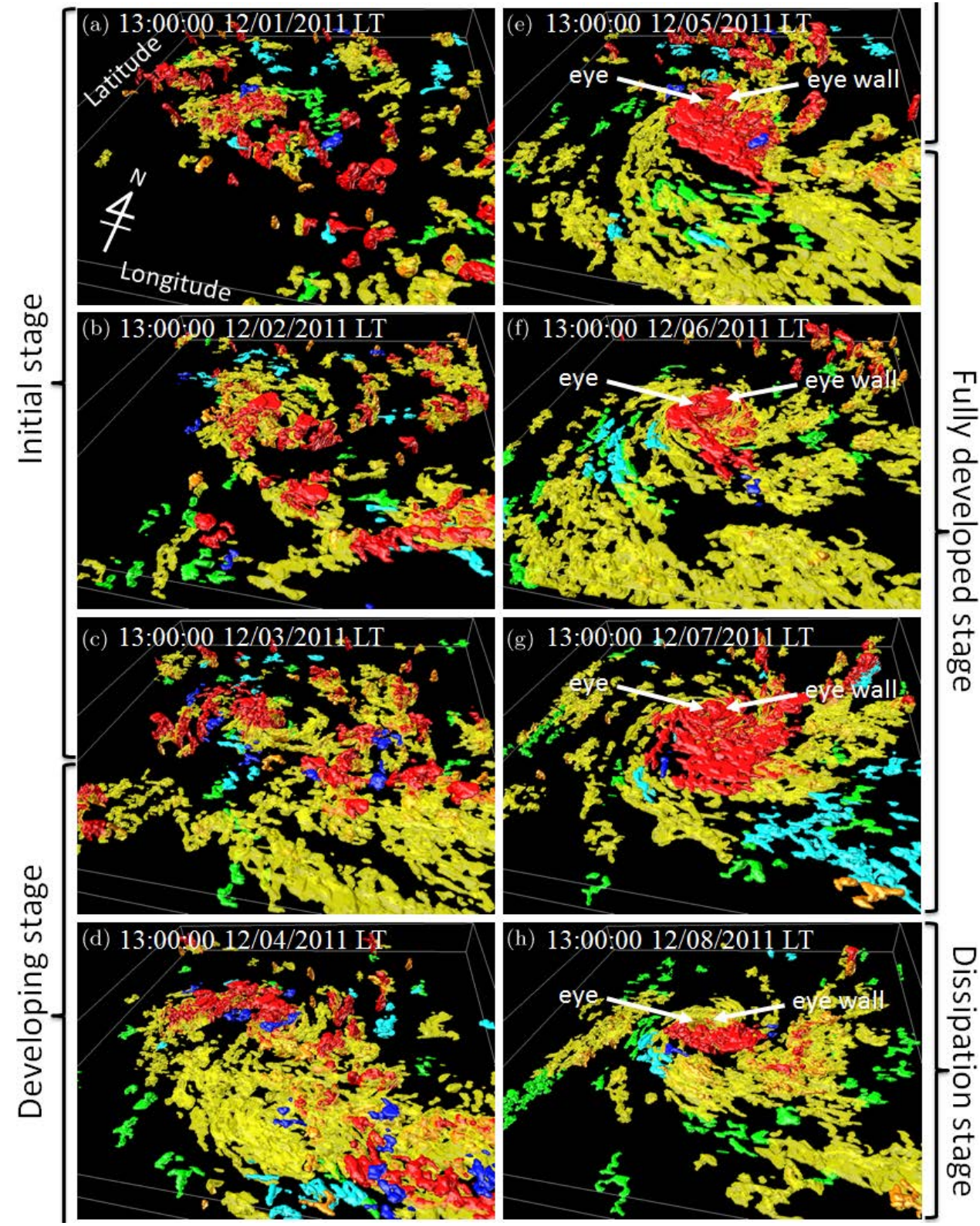

Fig. 9. Visualization results of temporal change of the tropical cyclone.

is called a tropical cyclone. In the fully developed stage, the size of the tropical cyclone is developed to the maximum (Figs. 9(e)-9(g)). In the dissipation stage, the size of the tropical cyclone, especially the size of cumulonimbus, becomes smaller (Fig. 9(h)). 


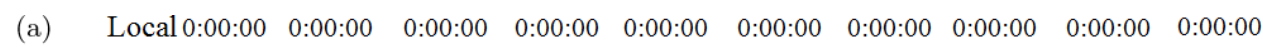
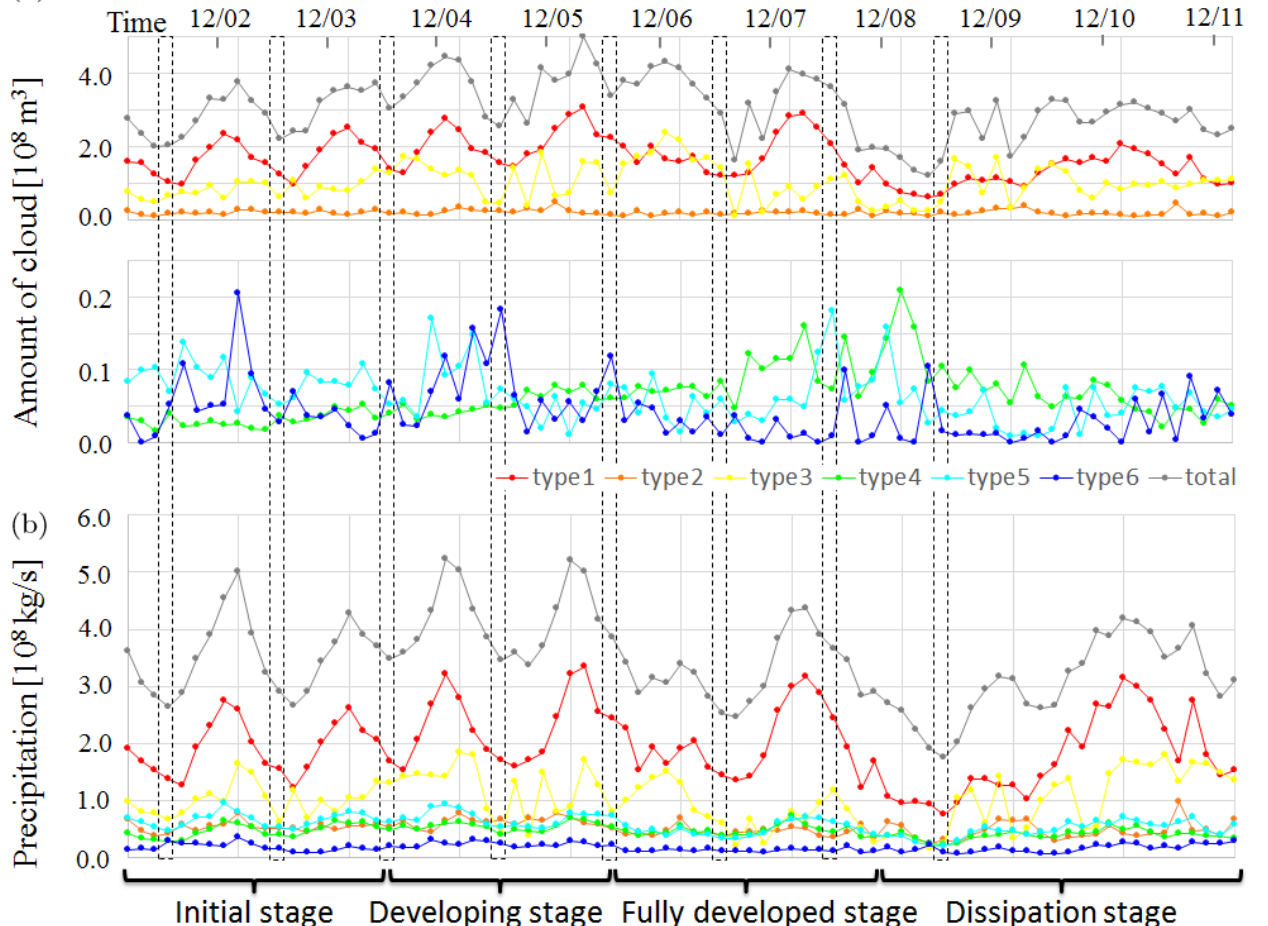

Fig. 10. Temporal change of cloud type and precipitation of the tropical cyclone. The Periods with dotted line corresponds to visualization results (Fig. 9).

We analyze cloud type and precipitation during the initial stage to the dissipation stage of the tropical cyclone. The relationship between cloud type and precipitation in the tropical cyclone from December 1 to 11, 2011 LT are shown in Figs.10(a) and10(b), respectively. From these results, the following facts are found out.

- Cumulonimbus is dominant with respect to both the amount of cloud and precipitation. The development of cumulonimbus is repeated in a one-day cycle. The peak of amount of cloud and precipitation is 3:00 LT, and the lesser is around 15:00 LT.

- Cumulonimbus gradually increases from the initial to the fully developed stage and decreases in the dissipation stage.

- Temporal change of total precipitation also has an one-day cycle and corresponds to the amount of cloud.

They are almost in agreement with the paper based on observational data. $\frac{18}{18}$ Furthermore, we clarified the following points as the unique facts of the proposed method: 
- Low clouds increase as tropical cyclone develops and decreases with decline.

- Opposite to this, high clouds decrease as tropical cyclone develops and increases with decline.

- These facts are supportive that tropical cyclones grow while capturing low clouds and decline while releasing high clouds.

\section{Conclusion}

In this study, we proposed a new extraction and classification method to intuitively understand simulated 3D clouds from cloud-resolving atmospheric simulation data. Extracted clouds are classified into six types using their altitude and vertical flow. As a result, we succeeded in visualizing the $3 \mathrm{D}$ configuration and their temporal changes during atmospheric events such as a developing cumulonimbus and a tropical cyclone. The proposed method is beneficial for intuitively understanding atmospheric phenomena.

We used lower temporal resolution data of $3 \mathrm{~h}$ in two case studies, however, using higher temporal resolution data will make tracking of individual clouds possible. In that case, it is predicted that storing and processing of dataset with enormous number of steps becomes a bottleneck. In situ processing which performs cloud extraction, tracking and visualization while simulating on a supercomputer will be key technology.

\section{Acknowledgments}

We are grateful to Drs. K. Oouchi, T. Miyakawa and M. Nakano and the NICAM team for their discussion, data production and model development. This work is supported by KAKENHI (26700010) Grant-in-Aid for Young Scientists (A) and KAKENHI (16K13885) Grant-in-Aid for Challenging Exploratory Research.

\section{References}

1. Ohfuchi W., Nakamura H., Yoshioka, M. K., Enomoto T., Takaya K., Peng X., Yamane S., Nishimura T., Kurihara Y., Ninomiya K., 10-km Mesh meso-scale resolving simulations of the global atmosphere on the earth simulator - preliminary outcomes of AFES (AGCM for the Earth Simulator), J. Earth Simul. 1:8-34, 2004.

2. Tomita S., Sato M., A new dynamical framework of nonhydrostatic global model using the icosahedral grid, Fluid Dyn. Res 34:357-400, 2004.

3. Sato M., Tomita H., Yashiro H., Miura H., Kodama C., Seiki T., Noda A. T., Yamada Y., Goto D., Sawada M., Miyoshi T., Niwa Y., Hara M., Ohno T., Iga S., Arakawa T., Inoue T., Kubokawa H., The Non-hydrostatic icosahedral atmospheric model: Description and development, Prog. Earth Planet. Sci. 1:1-32, 2014.

4. Miura H., Satoh M., Nasuno T., Noda A. T., Oouchi K., A madden-julien oscillation event realistically simulated by a global cloud-resolving model, Science 318:1763$1765,2007$.

5. Nakano M., Sawada M., Nasuno T., Satoh M., Intraseasonal variability and tropical cyclonenesis in the western north pacific simulated by a global nonhydrostatic atmospheric model, Geophys. Res. Lett. 42:565-571, 2015. 
6. Karlsson K.-G., Development of an operational cloud classification model, Int. J. Remote Sens 10:687-693, 1989.

7. Macias-Macias M., Garcia-Orellana C. J., Gonzalez-Velasco H., Gallardo-Caballero R., ICA and GA feature extraction and selection for cloud classification, Singh S., Singh M., Apte C., Perner P. (eds.) Int. Conf. Advances in Pattern Recognition 2005, Lecture Notes on Computer Science 3686, pp. 488-496. Springer, Heidelberg, 2005.

8. Zibert M. I., Derrien M., Le Gleau H., Automatic cloud classification by supervised learning on SEVIRI data using support vector machines method, The 2005 EUMETSAT Meteorological Satellite Conference, pp. 188-192, Dubrovnik, Croatia, 19-23 September 2005.

9. Kazantzidis R., Tzoumanikas P., Bais A. F., Fotopoulos S., Economou G., Equipment and methodologies for cloud detection and classification: A review, Sol. Energy 95:392-430, 2012.

10. Heinle A., Macke A. and Srivastav A., Automatic cloud classification of whole sky images, Atmos. Meas. Tech. 3:557-567, 2010.

11. Liu S., Zhang Z., Mei X., Ground-based cloud classification using weighted local binary patterns, J. Appl. Remote Sens 9:095062, 2015.

12. Matsuoka D., Oouchi K., 3-Dimensional Classification and Visualization of Clouds Simulated by Cloud-Resolving Atmospheric General Circulation Model, L. Zhang et al. eds: AsiaSim2016/SCS AutumnSim 2016, Part III CCIS 645:57-67, 2016.

13. The Ten Cloud Types, http://www.weathergamut.com/2011/10/14/cloud-nine/

14. Sinkevich A. A., Krauss T. W., Stepanenko V. D., Dovgalyuk Y. A., Veremey N. E., Krov A. B., Pivovarova L. V., Study of dynamics of the cumulonimbus anvil of large vertical extent, Russ. Meteorol. Hydrol. 34:775-783, 2010.

15. Mori S., Hamada J., Tauhid Y. I., Yamanaka M. D., Okamoto N., Murata F., Sakurai N., Hashiguchi H., Sribimawati T., Diurnal Land-Sea Rainfall Peak Migration Over Sumatera Island, Indonesian Maritime Continent, Observed by TRMM Satellite and Intensive Rawinsonde Soundings, Mon. Weather Rev. 132:2021-2039, 2004.

16. Houze R. A., Clouds in tropical cyclones, Mon. Weather Rev. 138:293-344, 2010.

17. Oouchi K., Fudeyasu H. (eds.), Cyclones: Formation, Triggers and Control, NOVA Publishers, New York, 2012.

18. Lepperta K. D., Cecil D. J., Tropical Cyclone Diurnal Cycle as Observed by TRMM, Mon. Weather Rev. 144:2021-2039, 2004. 